\title{
The role of extracellular vesicles in acquisition of resistance to therapy in glioblastomas
}

\author{
Anudeep Yekula', Abigail Taylor², Alexandra Beecroft ${ }^{2}$, Keiko M. Kang'1, Julia L. Small'1, Koushik \\ Muralidharan', Zachary Rosh ${ }^{1}$, Bob S. Carter ${ }^{1}$, Leonora Balaj ${ }^{1}$ \\ 'Department of Neurosurgery, Massachusetts General Hospital, Boston, MA 02114, USA. \\ ${ }^{2}$ Brigham Young University, Provo, UT 84602, USA.
}

Correspondence to: Dr. Bob S. Carter, Department of Neurosurgery, Massachusetts General Hospital, 55 Fruit St., Boston, MA 02114, USA. E-mail: bcarter@mgh.harvard.edu; Dr. Leonora Balaj, Department of Neurosurgery, Massachusetts General Hospital, 185 Cambridge St., Boston, MA 02114, USA. E-mail: balaj.leonora@mgh.harvard.edu

\begin{abstract}
How to cite this article: Yekula A, Taylor A, Beecroft A, Kang KM, Small JL, Muralidharan K, Rosh Z, Carter BS, Balaj L. The role of extracellular vesicles in acquisition of resistance to therapy in glioblastomas. Cancer Drug Resist 2021;4:1-16.

http://dx.doi.org/10.20517/cdr.2020.61
\end{abstract}

Received: 6 Aug 2020 First Decision: 17 Sep 2020 Revised: 5 Oct 2020 Accepted: 21 Oct 2020 Available online: 19 Mar 2021

Academic Editor: Joanna Kopecka Copy Editor: Cai-Hong Wang Production Editor: Jing Yu

\begin{abstract}
Glioblastoma (GBM) is the most aggressive primary brain tumor with a median survival of 15 months despite standard care therapy consisting of maximal surgical debulking, followed by radiation therapy with concurrent and adjuvant temozolomide treatment. The natural history of GBM is characterized by inevitable recurrence with patients dying from increasingly resistant tumor regrowth after therapy. Several mechanisms including inter- and intratumoral heterogeneity, the evolution of therapy-resistant clonal subpopulations, reacquisition of stemness in glioblastoma stem cells, multiple drug efflux mechanisms, the tumor-promoting microenvironment, metabolic adaptations, and enhanced repair of drug-induced DNA damage have been implicated in therapy failure. Extracellular vesicles (EVs) have emerged as crucial mediators in the maintenance and establishment of GBM. Multiple seminal studies have uncovered the multi-dynamic role of EVs in the acquisition of drug resistance. Mechanisms include EV-mediated cargo transfer and EVs functioning as drug efflux channels and decoys for antibody-based therapies. In this review, we discuss the various mechanisms of therapy resistance in GBM, highlighting the emerging role of EV-orchestrated drug resistance. Understanding the landscape of GBM resistance is critical in devising novel therapeutic approaches to fight this deadly disease.
\end{abstract}

Keywords: Glioblastoma, resistance, extracellular vesicles, temozolomide, radiation

\footnotetext{
(ㄷ) ( $)$

(C) The Author(s) 2021. Open Access This article is licensed under a Creative Commons Attribution 4.0 International License (https://creativecommons.org/licenses/by/4.0/), which permits unrestricted use, sharing, adaptation, distribution and reproduction in any medium or format, for any purpose, even commercially, as long as you give appropriate credit to the original author(s) and the source, provide a link to the Creative Commons license, and indicate if changes were made.
}

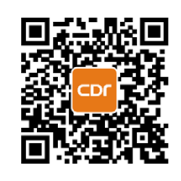




\section{INTRODUCTION}

Glioblastoma (GBM) is the most common primary brain tumor with a median survival of 15 months despite therapy ${ }^{[1]}$. The standard of care at initial diagnosis includes maximal surgical resection of the tumor followed by radiation therapy with concurrent and adjuvant temozolomide (TMZ) treatment ${ }^{[2,3]}$. The natural history of GBM is characterized by inevitable recurrence in 6-9 months after the initiation of therapy. GBMs are notorious for their ability to evade therapy and recur. Several researchers have focused their efforts on delineating the mechanisms underlying recurrence in GBM, with a special focus on therapy resistance. Multiple synergistic factors have been implicated in recurrence of GBM including inter- and intratumoral heterogeneity, the evolution of therapy-resistant clonal subpopulations, reacquisition of stemness in glioblastoma stem cells (GSCs), the impenetrable nature of the blood-brain barrier (BBB), multiple drug efflux mechanisms, the tumor-promoting microenvironment, metabolic adaptations, enhanced repair of drug-induced DNA damage, and tunneling tumor nanotubes ${ }^{[4]}$. Additionally, molecular factors such as TP53 mutation, MIB-1 labeling, and O-6-methylguanine-DNA methyltransferase have been correlated with GBM recurrence ${ }^{[5,6]}$. Management of recurrent GBM has been challenging with treatment options ranging from re-resection, re-radiation, systemic chemotherapy, immunotherapy, or a combination of these ${ }^{[7]}$. However, none of these strategies have proven beneficial. The choice of therapy is individualized depending on the time since diagnosis, radiological pattern, prior therapies, and, most importantly, the general and neurological function of the patient. Patients with recurrent GBM have a median survival of only 3-6 months ${ }^{[8]}$.

A series of seminal studies has uncovered the role of membrane-bound nanoparticles called extracellular vesicles (EVs) in the development of resistance in GBM. EVs are conduits for communication between cells, transporting molecular cargo including proteins, nucleic acids, and lipids from cell to cell ${ }^{[9,10]}$. The transfer of molecules facilitates tumor progression, angiogenesis, immune tolerance, modification of tumor metabolism, metastasis, invasion, and evasion of cell death ${ }^{[9,11-13]}$. Novel mechanisms such as EV-mediated drug efflux; EVs functioning as drug decoys; and EV-mediated transfer of functional mRNAs, miRNAs, long non-coding RNAs, spliceosomes, drug efflux pumps, and other resistance-acquiring products have been implicated in resistance to chemoradiation ${ }^{[12]}$. In this review, we explore the role of EV-orchestrated therapy in the global landscape of GBM therapeutic resistance. We emphasize the role of minimally invasive EV-based liquid biopsy platforms for monitoring recurrence, tumor evolution, and response to therapy $^{[14,15]}$.

\section{MECHANISMS OF THERAPY RESISTANCE IN GBM}

Therapeutic resistance remains a major barrier to the successful management of GBM. Multiple synergistic mechanisms have been described to characterize the ability of GBM to evade therapy. Understanding the cellular and molecular mechanisms underlying GBM therapy resistance is critical for the management of this deadly cancer.

\section{INTERTUMORAL, INTRATUMORAL HETEROGENEITY, AND CLONAL EVOLUTION}

Elegant sequencing studies in the past decade have unveiled the heterogeneous genomic and transcriptomic landscape of $\mathrm{GBM}^{[16-18]}$. At the genomic level, GBMs are broadly classified as IDH-wildtype or IDH-mutant. Additional genetic alterations including but not limited to epidermal growth factor receptor (EGFR) overexpression, phosphate and tensin homolog (PTEN) mutations, p53 mutations, and loss of chromosome 10q have been implicated. At transcriptomic levels, GBMs are categorized as proneural (harboring TP53, PDGFRA, and IDH mutations), classical (harboring EGFR mutations), or mesenchymal (NF1 mutations). Despite this heterogeneity, most tumors were found to harbor alterations in core oncogenic pathways: the tumor protein p53 pathway, the receptor tyrosine kinase/Ras/phosphoinositide 3-kinase signaling pathway, and the retinoblastoma pathway ${ }^{[19]}$. Additionally, recent single-cell sequencing studies have provided 
comprehensive evidence of the intratumor heterogeneity of GBM. Neftel et al ${ }^{[20]}$ demonstrated the presence of four specialized transcriptional subclones with varying rates of proliferation and transition within each tumor. Specifically, they identified neural-progenitor-like cells (NPC-like) with CDK4 amplifications, oligodendrocyte-progenitor-like cells (OPC-like) with PDGFRA amplifications, astrocyte-like cells (AClike) with EGFR aberrations, and mesenchymal-like cells (MES-like) with Chr5q deletions and NF1 alterations.

Currently available therapies are not tailored to each subclone, which leads to the selection of resistant and/ or untargeted subclones of cells. These selected resistant/untreated cells that have survived therapy due to their genetic makeup accumulate additional mutations and evolve into aggressive recurrent tumors ${ }^{[21-24]}$. This evolution of tumors can be compared to the Darwinian process of clonal selection ${ }^{[25]}$. Additionally, these subclones within recurrent tumors maintain differential drug resistance profiles ${ }^{[26]}$. Only $45 \%$ of mutations are shared between the primary and the recurrent tumor, with the dominant clone at recurrence being significantly different from the dominant clone at diagnosis ${ }^{[21,27]}$. This selection of clonal subtypes and accumulation of alterations can produce diverse clonal populations with series of parallel expansions within each tumor ${ }^{[23]}$. The spatiotemporal divergence in recurrent tumors also occurs at epigenomic and transcriptomic levels ${ }^{[23,24,28]}$. This evolutionarily divergent clonal selection is the crux of therapy failure and development of resistance. In this regard, the current practice of selecting a therapeutic option for a recurrent tumor based on the characteristics of the primary tumor may not be appropriate ${ }^{[23]}$. Therapeutic strategies should be tailored to the clonal subpopulations in order to radically target all/most cell types and prevent/minimize the selection of aggressive tumor subclones. This highlights the need for the development of multimodal poly-therapeutic strategies as opposed to monotherapies ${ }^{[2]]}$.

\section{GLIOBLASTOMA STEM CELLS}

GSCs represent a group of self-sustained cells that have attained a mutational profile capable of tumorigenesis due to their ability to proliferate and self-renew ${ }^{[30]}$. Multiple studies have demonstrated the tumorigenic properties of GSCs ${ }^{[30,31]}$. These cells are classically identified based on the presence of cell surface markers such as $\mathrm{CD} 133, \mathrm{CD} 44$, and $\mathrm{L} 1$ cell adhesion molecules (L1CAM) ${ }^{[32,33]}$. Furthermore, GSCs modulate the tumor microenvironment supporting tumor growth and proliferation ${ }^{[12]}$. Despite therapy, these GSCs maintain stemness, survive, and proliferate to produce a recurrent tumor. Recent studies have demonstrated that the GSCs in recurrent GBM differ from the GSCs that initiated and maintained the primary tumor. The GSCs of recurrent tumors are more aggressive, display altered surface marker profiles (loss of CD133 and gain of CD15, BMI1, and SOX2), and correlate with shorter survival of patients in a recurrent setting ${ }^{[34-36]}$.

Recent studies have recapitulated the existence of quiescent and active cancer stem cells ${ }^{[37]}$. Researchers have hypothesized that chemoradiation targets rapidly dividing active cancer stem cells while the quiescent cancer stem cells survive chemoradiation, which eventually recapitulates the tumor ${ }^{[37,38]}$. Additionally, CD133+ GSCs have demonstrated chemoresistance and radioresistance. In GSCs, TMZ chemoresistance is mediated by increased expression of anti-apoptotic genes, MGMT-mediated DNA repair mechanisms, and drug efflux transporters ${ }^{[39]}$. Radioresistance is mediated through activation of DNA damage checkpoint and repair of radiation-induced DNA damage by arresting cell cycle ${ }^{[40]}$. GSCs acquire therapeutic resistance following repeated chemoradiation ${ }^{[35,41]}$. Recent studies have unveiled pathways involved in GSC maintenance, survival, self-renewal, and proliferation. These have been comprehensively elaborated in a recent review ${ }^{[42]}$. Targeting these GSCs and their associated pathways has been explored but limited in success $^{[43]}$. Curtailing the GSC populations will be critical in developing therapeutic strategies to combat recurrence. 


\section{BBB AND DRUG EFFLUX MECHANISMS}

Achievement of therapeutic concentrations of chemotherapeutic drugs in the CNS has been a major challenge in the management of GBM. The BBB, which separates the lumen of cerebral blood vessels from the brain parenchyma, stringently regulates the entry of circulating toxins, macromolecules, and inflammatory cells. The tight junctions between the endothelial cells of CNS blood vessels in addition to the supportive pericytes and astrocytes maintain the integrity of the $\mathrm{BBB}^{[44]}$. GBM disrupts the integrity of the $\mathrm{BBB}$, and this endothelium in contact with the GBM is referred to as the blood-tumor barrier (BTB). In GBM, the BTB has a heterogeneous permeability owing to local anatomic invasion, downregulation of tight junction proteins, upregulation of transporter proteins, and angiogenesis. The BTB is also characterized by aberrant pericyte distribution and the loss of astrocytic endfeet and neuronal connections ${ }^{[45]}$. Furthermore, the BBB is reinforced by efflux pumps including ATP-binding cassette efflux transporters, P-glycoprotein (P-gp), multidrug resistance protein (MRP), and breast cancer resistance protein, which actively transport out substrates ${ }^{[46-49]}$. Interestingly, these drug efflux transporters were also described to reduce intracellular drug accumulation in GBM cells ${ }^{[46,50]}$. The BBB/BTB in conjunction with BBB associated efflux transporters functions synergistically as tumor-promoting adaptive mechanisms to selectively permit the transport of tumor-supporting substances while limiting the entry of antitumor substances ${ }^{[4]}$. Although "leaky", the disrupted BBB in GBM paradoxically limits the achievement of therapeutic concentrations of chemotherapeutic drugs. Additionally, altered cell adhesion molecular profiles of BBB endothelial cells have been shown to limit the transmigration of adoptively transferred $\mathrm{T}$ cells and chimeric antigen receptor (CAR) T cells, posing a substantial challenge ${ }^{[45]}$.

Several strategies have been attempted to target the BBB to enhance the delivery of chemotherapeutic agents: molecular approaches such as the use of endogenous influx transporter-mediated drug delivery; invasive approaches such as convection-enhanced delivery by direct injection, intrathecal, intraventricular injections, and implantation of wafers, gels, and microchips to bypass the BBB; and physical approaches such as magnetic resonance-guided focused ultrasound-mediated disruption of the BBB. These modalities have their drawbacks and offer limited benefit ${ }^{[45]}$. Co-administration of chemotherapeutic drugs in conjunction with inhibitors of BBB-associated efflux transporters have shown promise ${ }^{[51-53]}$. Unfortunately, all these modalities have technical limitations, inadequate CNS penetrance, systemic toxicities, and limited efficacy $^{[4,54]}$.

\section{GLIOBLASTOMA TUMOR MICROENVIRONMENT}

The GBM tumor microenvironment consists of glial cells (astrocytes, oligodendrocytes, ependymal cells, and microglia) and invading immune cells (macrophages, monocytes, and lymphocytes). GBM hijacks the tumor microenvironment and modifies it into a tumor-promoting environment ${ }^{[55]}$. These adaptations allow the tumors to survive various chemotherapeutic insults and proliferate. Researchers have attempted to harness the potential of immune cells of the TME to generate anti-tumor responses. Immunotherapy for GBM includes checkpoint inhibitors, CAR-T therapy, vaccines, and viral therapy ${ }^{[55,56]}$. In addition to challenges such as intra-tumoral and intratumoral heterogeneity, the tumor microenvironment provides unique hurdles to the success of immunotherapy. Immunosurveillance of the CNS is inherently adapted to maintain neuronal function and minimize non-specific immune responses. Additionally, GBM generates an immunosuppressive microenvironment by secreting soluble factors, interleukins, prostaglandins, and EVs to induce a tumor supportive M2 phenotype in tumor-associated myeloid cells (microglia, monocytes, and macrophages), induce $\mathrm{T}$ cell dysfunction, and suppress NK cell activity ${ }^{[12,56]}$. The limitations of the immune response in the CNS and the highly immunosuppressive nature of GBM limit the effectiveness of the antitumor response induced by immunotherapeutic agents. 


\section{METABOLIC ADAPTATIONS}

Metabolic adaptations of GBM are intrinsic to the growth and proliferation. Detailed metabolic alterations in GBM and specific pathways implicated in therapy resistance are outlined in recent reviews ${ }^{[57,58]}$. Targeting metabolic pathways governing GBMs adaptations has been of interest over the past decade ${ }^{[58]}$. However, there is growing evidence supporting the evolution of resistance mechanisms ${ }^{[59]}$. Rapidly growing tumors such as GBM are characterized by intratumoral hypoxia and necrosis due to deficiencies in $\mathrm{O}_{2}$ delivery and availability. GBM cells adapt to the hypoxic microenvironments through the activation of hypoxiainducible factors promoting angiogenesis, induction of stem-like phenotype, chemoresistance, and motility ${ }^{[60]}$. Hypoxia facilitates anaerobic glycolysis and the pentose phosphate pathway, which provide survival advantage ${ }^{[61,62]}$. Hypoxic environments have a reduced concentration of therapeutic agents due to limited vascular supply and upregulation of drug efflux ${ }^{[63,64]}$. Emerging evidence suggests the presence of metabolically quiescent cells which could potentially be additional mediators of therapy resistance ${ }^{[65,66]}$. Interestingly, studies have highlighted the role of unfolded protein response-based stress effects in tumorprotective effects and TMZ chemoresistance in gliomas ${ }^{[67]}$. Thus, understanding the multidimensional role of hypoxia, angiogenesis, and metabolic alterations in resistance to therapy will provide attractive therapeutic options.

\section{DRUG DAMAGE REPAIR}

Tumor cells depend excessively on DNA repair mechanisms to help them cope with exacerbated DNA damage resulting from increased metabolism, replication, and mitotic stress. Alkylating agents (TMZ) and ionizing radiation primarily induce DNA damage. Double-stranded breaks (DSB) inflicted by radiation are repaired by homologous recombination and non-homologous end-joining, while single-stranded breaks $(\mathrm{SSB})$ are repaired by base excision repair (BER) and single-stranded repair mechanisms ${ }^{[68,69]}$. TMZ induces the formation of N3-methyladenine (N3-meA) and N7-methylguanine (N7-meG), cytotoxic lesions which are primarily repaired by BER, and O6-methylguanine (O6-meG), a cytotoxic lesion which is managed by O6-methylguanine-DNA methyltransferase $(\mathrm{MGMT})^{[70]}$. Epigenetic silencing of MGMT promoter by methylation reduces tumoral DNA repair capacity and is associated with increased TMZ sensitivity ${ }^{[71]}$. In the absence of MGMT, O6-meG mispairs with cytosine or thymine. Mismatch repair machinery (MMR) recognizes the mispairs and leaves the O6-meG intact, leading to SSBs and DSBs ${ }^{[2,72]}$. Efficient DNA repair mechanisms and the loss of MMR activity have been implicated in GBM resistance and recurrence. Several therapeutic strategies aimed at the DNA repair axis via MGMT transcriptional regulation, MGMT silencing, and inhibition of DNA repair mechanisms are under exploration and provide attractive therapeutic targets ${ }^{[73,74]}$.

\section{TUMOR MICROTUBULES}

A recent study by Osswald and colleagues showed that GBM tumor cells form a functional network by extending ultralong membrane protrusions called tumor microtubules (TMs) to connect and communicate with neighboring tumor cells. TMs are implicated in tumor cell invasion, proliferation, interconnection, and radioresistance. Cytotoxic effects of radiation are largely mediated by an increase in intracellular calcium. TM-connected tumor cells have displayed resistance to the cytotoxic effects of radiation by maintaining calcium homeostasis by network integration. Additionally, TMs provide a means of effective distribution of small molecules among other cells of the network ${ }^{[4,75]}$. Pharmacological targeting of TM formation should be explored for the management of treatment-resistant brain tumors.

\section{ROLE OF EXTRACELLULAR VESICLES IN THERAPY RESISTANCE}

Extracellular vesicles have attracted widespread interest over the past decade for their role in modulating tumor microenvironment to promote tumor growth, proliferation, angiogenesis, therapy resistance, invasion, and evasion of immune surveillance. We elaborated on the multidimensional role of EVs in the 


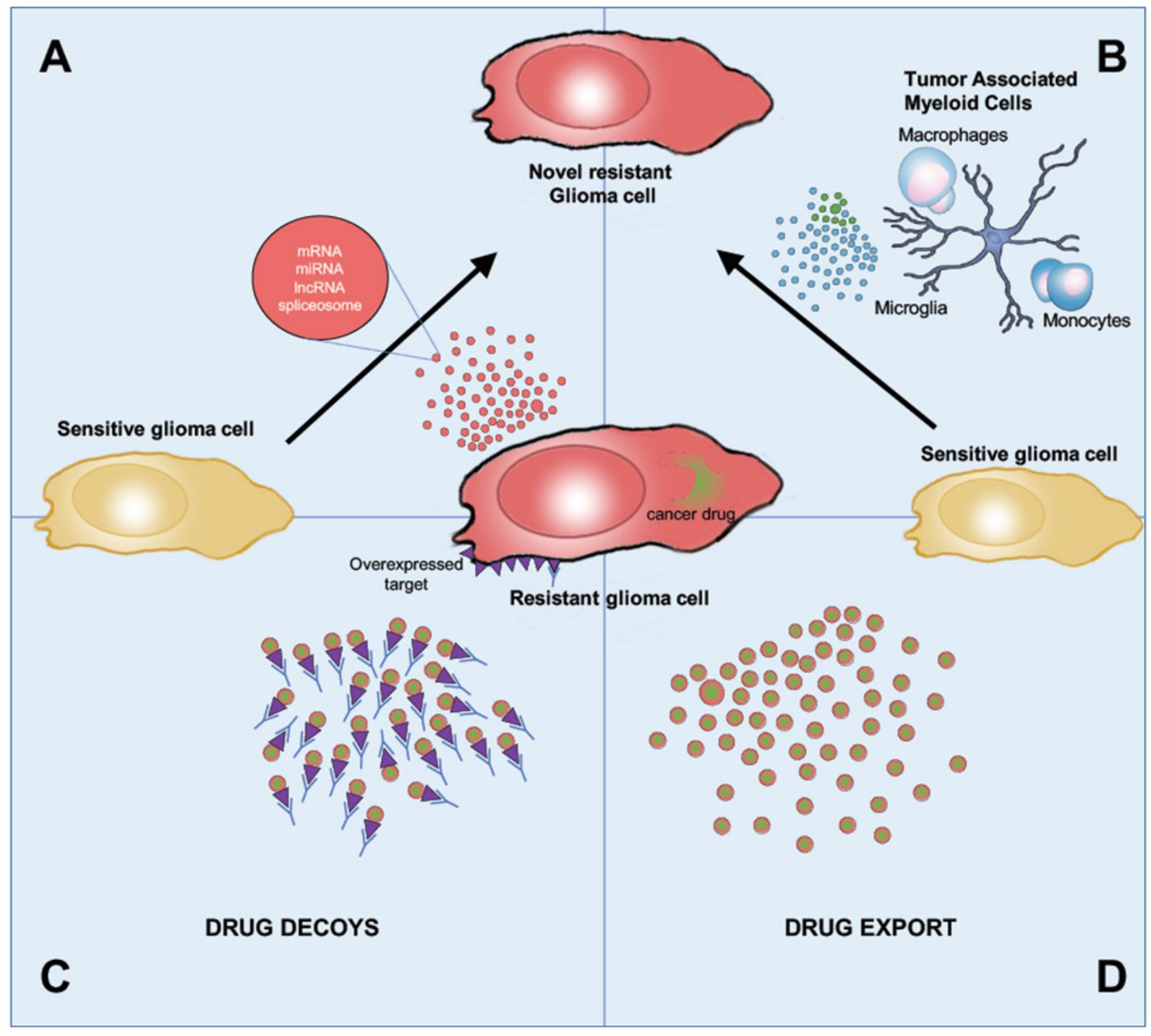

Figure 1. Overview of EV-mediated mechanisms of drug resistance. EVs derived from (A) resistant tumor cells and (B) tumor supporting cells transfer genomic and proteomic cargo to glioma treatment sensitive cells, which enhances their acquisition of a resistant phenotype; (C) EVs also function as decoys for antibody-based therapies, leading to the sequestration of anticancer antibodies; (D) EVs package and export drugs out of the cells, reducing its intracellular concentration

tumor microenvironment in our recent review ${ }^{[12]}$. EV-mediated therapy resistance is yet another adaptation of GBM to overcome therapy. Multiple EV-mediated mechanisms of therapy resistance have been described across the literature in the context of numerous systemic cancers including breast, prostate, lung, renal, ovarian, hematologic, pancreatic, gastric, and brain cancers ${ }^{[76]}$. The major EV-mediated mechanisms of acquisition of therapy resistance are depicted in Figure 1.

\section{GBM-MEDIATED EV TRANSFER}

Multiple studies across several systemic cancers have shown that EV-mediated transfer of functional cargo (mRNAs, miRNAs, long non-coding RNA (lncRNA), and proteins) induces drug resistance in drugsensitive cancer cells ${ }^{[76]}$. The transferred functional molecules upsurge drug efflux ${ }^{[77-80]}$, enhance drug metabolism/inactivation ${ }^{[81]}$, activate anti-apoptotic and tumor-promoting pathways ${ }^{[82-88]}$, elicit downstream changes in signal transduction and gene expression ${ }^{[89-95]}$, and promote epithelial-to-mesenchymal transition ${ }^{[96-99]}$ to favor a resistant phenotype. 
GBM cells actively modulate the composition of EVs in response to chemotherapy and radiation ${ }^{[100,101]}$. The resistant GBM cells produce specialized EVs with cargo capable of inducing a resistant phenotype in the recipient sensitive cells. EV-mediated transfer of multiple genomic and proteomic cargo including mRNA, miRNA, lncRNA, spliceosomes, and proteins have been reported in the context of TMZ and radioresistance in $\mathrm{GBM}^{[102-108]}$. The EV cargo causes a range of downstream effects in the recipient sensitive cells. Transfer of transcripts of DNA repair enzymes such as alkyl purine-DNA-N-glycosylase (APNG) and O(6)methylguanine DNA methyltransferase (MGMT) causes increased DNA repair capability in the recipient cells ${ }^{[109,110]}$. Uptake of the lncRNA $\operatorname{lncSBF} 2$-AS was shown to enhance DNA damage repair by upregulating the lncSBF2-AS1-miR-151a-3p-XRCC4 DNA repair axis ${ }^{[11]}$. EV-mediated transfer of miRNAs has been demonstrated to activate anti-apoptotic pathways, enhancing GBM cell proliferation in sensitive GBM cells in response to TMZ and radiotherapy ${ }^{[112-114]}$.

\section{EVS DERIVED FROM CELLS OF THE TUMOR MICROENVIRONMENT}

In addition to the transfer of cargo and drug resistance mechanisms spread from resistant cells to sensitive cells, stromal cells and immune cells were also implicated in EV-mediated resistance transfer. Specifically, EVs derived from cancer-associated fibroblasts ${ }^{[115-118]}$, cancer-associated adipocytes ${ }^{[118]}$, and tumor-associated macrophages ${ }^{[119,120]}$ in the tumor microenvironment have been shown to impart drug resistance in sensitive cells via the transfer of functional cargo. EVs derived from GBM associated macrophages and GBM associated astrocytes were also implicated in the development of TMZ resistance and radioresistance ${ }^{[109,121]}$.

\section{EVS AS DRUG EFFLUX CHANNELS}

Cancer cells have been shown to package and export chemotherapeutic agents in EVs. Recent studies in ovarian and pancreatic cancer-derived EVs demonstrate the presence of drug efflux transporters such as MRP and P-gp. This localization of drug efflux pumps enhances the uptake of drugs into the vesicles for eventual release ${ }^{[122,123]}$. The presence of transporters in reverse orientation on the surface of EVs, leading to drug import into EVs, further validated this mechanism ${ }^{[76,124]}$. Studies in breast cancer have shown that cell lines resistant to mitoxantrone confine the drug in EV-like structures within the cell near the cell-cell attachment regions. These EV-like structures contain drug efflux transporters that mediate intravesicular drug accumulation. However, the authors did not investigate the release of these EV-like structures ${ }^{[125]}$. This export of chemotherapeutic agents via EVs leads to a reduction in the intracellular concentration of the drug, reducing its efficacy and promoting resistance.

\section{EVS FOR DECOYS AS ANTIBODY-BASED THERAPIES}

EVs released by cancer cells contain surface markers, which are often targets for antibody-based immunotherapies. Anticancer antibodies that target specific surface markers of interest have been demonstrated to bind to EVs containing the same surface markers. This leads to the neutralization of anticancer antibodies (anti-HER2 monoclonal antibody, trastuzumab ${ }^{[125]}$, and anti-CD20 monoclonal antibody, rituximab ${ }^{[79]}$ ) by competitive inhibition, thus reducing their bioavailability. This was supported by the detection of large quantities of monoclonal anti-cancer antibody-EV complexes ${ }^{[79,126]}$. Neutralization of anti-cancer antibodies by EVs has been shown to reduce the bioavailability of the drug by almost $50 \%{ }^{[79]}$. Elegant studies by Simon and colleagues identified bevacizumab (monoclonal anti-VEGF antibody) on the surface of EVs derived from glioma cells treated with bevacizumab but not on EVs derived from control cells. The formation of EV-bevacizumab complexes reduces the bioavailability and the efficacy of the antiangiogenic drug in vitro. Furthermore, inhibition of EV production increased the efficacy of the drug, validating the role of EVs as drug decoys. However, these mechanisms have not yet been validated in vivo and the downstream effects of EV-bevacizumab complexes are still unknown ${ }^{[127]}$. 
Detailed descriptions of studies describing EV-mediated mechanisms of EV resistance in GBM are outlined in Table 1. In summary, the transfer of these factors enhances therapy resistance by a reduction in intracellular concentration, inactivation of chemotherapeutic drugs, activation of antiapoptotic pathways, upregulation of tumor-promoting pathways, and promoting a switch to a mesenchymal phenotype. These phenomena can be considered additional ways of intercellular communication between resistant and sensitive cancer cells to overcome therapy. They provide insight into the tremendous capability of the GBM cells to dynamically modulate EV composition based on the noxious stimulus they are exposed to. This principle can be broadly applied to the multitude of stresses that cancer cells face, as well as their effective communication and coping mechanisms to overcome these hurdles. Interestingly, multiple studies showed that treating resistant cells with EVs carrying drug sensitizing cargo induced chemosensitivity in the recipient cells ${ }^{[128,129]}$, indicating the impact of transferred functional cargo and the potential role of EVs as drug delivery tools. Understanding the role of EVs in the acquisition of resistance is important to target EV-mediated escape mechanisms.

\section{ROLE OF EXTRACELLULAR VESICLES IN TRACKING THE EVOLUTION OF RESISTANCE}

In the dynamic setting of the GBM disease course, imaging modalities are limited in providing molecular information about the intratumoral heterogeneity and clonal evolution in the setting of therapy resistance. Liquid biopsy-based modalities have been explored as a potential way to provide information regarding the genomic, transcriptomic, and proteomic changes that occur in a tumor over the course of time and therapy ${ }^{[130-132]}$. Emerging evidence from several liquid biopsy studies showed higher quantities of resistance mediators can be detected in serum EVs of cancer patients who did not respond to chemotherapy compared to responders ${ }^{[77,81,133-135]}$. The dynamic role of liquid biopsy-based strategies in monitoring the course of GBM has been elaborated in recent reviews. The biomarker potential of EV-based mediators of GBM resistance described in Table 1 needs to be evaluated. This could provide an opportunity to longitudinally track the evolution of the tumor over disease course to strategize therapies based on the tumor's behavior in response to therapy. Furthermore, liquid biopsy may also have the potential to guide clinical trials to recruit patients with recurrent GBM based on the molecular state of the recurrent tumors rather than the treatment-naive primary tumor.

\section{CURRENT STATUS OF THERAPY}

In GBM, the development of resistance to treatment and thus disease recurrence and progression is a foregone conclusion. However, there are no standardized treatment paradigms for recurrent or progressive GBMs. Current treatment strategies for recurrent GBM use multimodal strategies that focus on the debulking of symptomatic tumors, the cytotoxic effects of radiation and chemotherapy, and targeted treatment with RTKs and immunotherapies ${ }^{[136-138]}$. However, each of these strategies has pitfalls that have averted durable disease control.

Reoperation for GBM can be helpful to confirm disease recurrence, sample the current molecular profile of the tumor, and relieve symptomatic mass effect. Resection allows for a reduction in clonal diversity within the tumor; however, less than $25 \%$ of patients undergo a second operation due to tumor location or poor prognostic factors such as low Karnofsky performance status ${ }^{[139]}$. In the event of reoperation, the extent of resection is a predictor for overall survival; however, a multicenter study of 503 patients undergoing reresection for recurrent GBM demonstrated that complete and near-complete ( $\geq 90 \%,<100 \%)$ extent of resection was reduced in reoperation as compared to initial operation ${ }^{[140]}$. There is evidence to suggest local strategies, such as carmustine wafers placed in the resection cavity, improve survival in patients with local recurrence; however, the adverse effects associated with wafer removal and their long-term benefits are still being evaluated ${ }^{[141]}$. Local control of the tumor in the surgical site has also been attempted using 5-aminolevulinic acid (5-ALA)-based photodynamic therapy, but with variable efficacy ${ }^{[142,143]}$. Radiation 
Table 1. Summary of studies describing EV-mediated mechanisms of EV resistance in GBM

\begin{tabular}{|c|c|c|c|c|c|}
\hline Author, year & Therapy & $\begin{array}{c}\text { Mechanism of EV } \\
\text { mediated resistance } \\
\text { transfer }\end{array}$ & $\begin{array}{l}\text { Genetic cargo } \\
\text { evaluated }\end{array}$ & Functional implication & $\begin{array}{l}\text { Validation: in } \\
\text { vitro/in vivo }\end{array}$ \\
\hline Zhang et al. ${ }^{[111]}, 2019$ & TMZ & $\begin{array}{l}\text { EV mediated cargo } \\
\text { transfer }\end{array}$ & $\begin{array}{l}\text { IncRNA, } \\
\text { IncSBF2-AS }\end{array}$ & $\begin{array}{l}\text { Enhanced DNA damage repair by } \\
\text { upregulating IncSBF2-AS1-miR- } \\
\text { 151a-3p-XRCC4 DNA repair axis }\end{array}$ & Both \\
\hline Yin et al. ${ }^{[112]}, 2019$ & $\mathrm{TMZ}$ & $\begin{array}{l}\text { EV mediated cargo } \\
\text { transfer }\end{array}$ & $\begin{array}{l}\text { miRNA, } \\
\text { miR-1238 }\end{array}$ & $\begin{array}{l}\text { Anti-apoptotic function by the } \\
\text { activation of EGFR-PI3K-Akt-mTOR } \\
\text { pathway }\end{array}$ & Both \\
\hline Chuang et al. ${ }^{[121]}, 2019$ & TMZ & $\begin{array}{l}\text { GBM associated } \\
\text { macrophage EV } \\
\text { mediated cargo transfer }\end{array}$ & $\begin{array}{l}\text { miRNA, } \\
\text { miR-21-5p }\end{array}$ & $\begin{array}{l}\text { Enhanced survival by modulating } \\
\text { tumor suppressor gene, PDCD4 } \\
\text { and enhancing STAT3/JAK } 2 \\
\text { pathway }\end{array}$ & Both \\
\hline Zeng et al. ${ }^{[104]}, 2017$ & TMZ & $\begin{array}{l}\text { EV mediated cargo } \\
\text { transfer from (PTPRZ1- } \\
\text { MET-ZM fusion positive } \\
\text { cells) }\end{array}$ & $\begin{array}{l}\text { Specific cargo not } \\
\text { identified }\end{array}$ & - & Both \\
\hline Munoz et al. ${ }^{[114]}, 2019$ & $\mathrm{TMZ}$ & $\begin{array}{l}\text { EV mediated cargo } \\
\text { transfer }\end{array}$ & $\begin{array}{l}\text { miRNAs, } \\
\text { miR-93, miR-193 }\end{array}$ & $\begin{array}{l}\text { Decrease cell cycling quiescence by } \\
\text { targeting Cyclin D1 }\end{array}$ & In vitro \\
\hline Yu et al. ${ }^{[109]}, 2018$ & $\mathrm{TMZ}$ & $\begin{array}{l}\text { GBM associated } \\
\text { Astrocyte EV mediated } \\
\text { cargo transfer }\end{array}$ & $\begin{array}{l}\text { mRNA, } \\
\text { MGMT }\end{array}$ & $\begin{array}{l}\text { Transfer of MGMT mRNA increases } \\
\text { DNA repair enzymes in recipient } \\
\text { cells }\end{array}$ & Both \\
\hline Shao et al. ${ }^{[110]}, 2015$ & TMZ & $\begin{array}{l}\text { EV mediated cargo } \\
\text { transfer }\end{array}$ & $\begin{array}{l}\text { mRNA, MGMT and } \\
\text { APNG }\end{array}$ & $\begin{array}{l}\text { Transfer of MGMT, APNG mRNA } \\
\text { increases DNA repair enzymes in } \\
\text { recipient cells }\end{array}$ & In vitro \\
\hline Pavlyukov et al. ${ }^{[105]}, 2018$ & $\begin{array}{l}\text { Radiation, } \\
\text { TMZ, } \\
\text { Cisplatin }\end{array}$ & $\begin{array}{l}\text { EV (Apoptotic) } \\
\text { mediated cargo transfer }\end{array}$ & $\begin{array}{l}\text { Spliceosome, } \\
\text { RBM11 }\end{array}$ & $\begin{array}{l}\text { RBM11 switches splicing of MDM4 } \\
\text { and Cyclin D1 }\end{array}$ & Both \\
\hline $\begin{array}{l}\text { André-Grégoire et al. }{ }^{[108]}, \\
2018\end{array}$ & $\mathrm{TMZ}$ & $\begin{array}{l}\text { EV mediated cargo } \\
\text { transfer }\end{array}$ & $\begin{array}{l}\text { Proteolytic and } \\
\text { mRNA processing } \\
\text { proteins, adhesion } \\
\text { related proteins }\end{array}$ & - & In vitro \\
\hline $\begin{array}{l}\text { Mrowczynski et al. }{ }^{[113]}, \\
2018\end{array}$ & Radiation & $\begin{array}{l}\text { EV mediated cargo } \\
\text { transfer }\end{array}$ & $\begin{array}{l}\text { Upregulated: } \\
\text { miRNA, miR-889 } \\
\text { mRNA, WWC1 } \\
\text { Downregulated: } \\
\text { miRNA, miR-365 }\end{array}$ & $\begin{array}{l}\text { Upregulated miR-889 (inhibits } \\
\text { DAB2IP expression), mRNA } \\
\text { WWC1, and downregulated miR- } \\
365 \text { (disinhibiting expression of } \\
\text { Cyclin-D1, BCL-2, and PI3K and } \\
\text { PTEN) increases radioresistance }\end{array}$ & Both \\
\hline Zhang et al. ${ }^{[102]}, 2020$ & Radiation & $\begin{array}{l}\text { GBM associated } \\
\text { macrophage EV } \\
\text { mediated cargo transfer }\end{array}$ & $\begin{array}{l}\text { miRNAs, miR-27a- } \\
\text { 3p, miR-22-3p and } \\
\text { miR-221-3p }\end{array}$ & $\begin{array}{l}\text { Promoted proneural to } \\
\text { mesenchymal transition by } \\
\text { targeting CHD7 pathway }\end{array}$ & Both \\
\hline Yue et al. ${ }^{[103]}, 2019$ & Radiation & $\begin{array}{l}\text { Hypoxia induced EV } \\
\text { mediated cargo transfer }\end{array}$ & miRNA, miR-301a & $\begin{array}{l}\text { Activates Wnt/ } \beta \text {-catenin Signalling } \\
\text { and inhibiting TCEAL7 }\end{array}$ & In vitro \\
\hline Dai et al. ${ }^{[107]}, 2019$ & Radiation & $\begin{array}{l}\text { EV mediated cargo } \\
\text { transfer from AHIF } \\
\text { positive cells }\end{array}$ & $\begin{array}{l}\text { Specific cargo not } \\
\text { identified }\end{array}$ & $\begin{array}{l}\text { AHIF-mediated p53 } \\
\text { downregulation and anti-apoptosis }\end{array}$ & In vitro \\
\hline $\begin{array}{l}\text { Ramakrishnan et al. }{ }^{[106]}, \\
2020\end{array}$ & Radiation & miRNA export & miRNA, miR-603 & $\begin{array}{l}\text { miR- } 603 \text { export causes de- } \\
\text { repression of IGF1, IGF1R and } \\
\text { MGMT leading to radioresistance } \\
\text { and TMZ resistance }\end{array}$ & Both \\
\hline Simon et al. ${ }^{[127]}, 2018$ & Bevacizumab & Decoys & - & $\begin{array}{l}\text { Reduced bioavailability of } \\
\text { bevacizumab }\end{array}$ & In vitro \\
\hline
\end{tabular}

AHIF: antisense transcript of hypoxia-inducible factor-1 $\alpha$; APNG: alkyl purine-DNA-N-glycosylase; EV: extracellular vesicles; GBM: glioblastoma; IGF1: insulin-like growth factor 1; IGF1R: insulin-like growth factor 1 receptor; MGMT: O(6)-methylguanine-DNA methyltransferase; TMZ: temozolomide

therapies, including stereotactic radiosurgery (i.e., high-dose radiation delivered in one dose), radiotherapy (i.e., fractions of radiation delivered over multiple doses), and brachytherapy (i.e., direct delivery of radiation treatment via implantable devices), have also been explored for the treatment of recurrent $\mathrm{GBM}^{[144-147]}$. However, these therapies are limited by maximal dose, radiation-induced toxicity, and risk of complications ${ }^{[136]}$. Additionally, GBMs can develop resistance to radiation therapies due to activation and adaptation of DNA repair pathways ${ }^{[42,148]}$. 
Systemic therapies, including chemotherapeutics, targeted agents, and immunotherapies have also had limited efficacy in recurrent GBM. Bevacizumab, an anti-angiogenic monoclonal antibody targeting vascular endothelial growth factor A (VEGFA), now often used as a first-line agent for recurrent GBMs, first showed promise for the treatment of recurrent GBM in 2009 and 2010, when a series of studies assessed its use in mono- and combination therapy regimens. Although recurrent GBMs demonstrate a radiographic response to bevacizumab, the drug is not associated with increased overall survival. However, many practicing clinicians regard its positive effects such as potential sparing of steroid dose and some neurologic improvement ${ }^{[149-152]}$. Resistance to bevacizumab and other anti-angiogenic agents may be due to the complex network and crosstalk between different RTKs ${ }^{[138]}$ Furthermore, GBMs are thought to escape angiogenic growth mechanisms via upregulation of hypoxic growth factors, tumor invasion, and necrosis ${ }^{[153,154]}$. Nitrosoureas, such as carmustine and lomustine, are often used as second-line agents for recurrent GBM; however, the development of resistance and toxicity profiles limit the applicability of these drugs $^{[153,155]}$. Patients with recurrent GBM can also be re-challenged with TMZ. Similar to nitrosoureas, this strategy often fails due to hypermutation or activation of DNA repair pathways which circumnavigate TMZ's mechanism of action ${ }^{[153]}$. Immunotherapy modalities including checkpoint inhibitor therapies, CAR-T cell therapy, and vaccine trials have been increasingly explored. The brain immunology and accompanying therapeutic targets are masterfully discussed in a recent review by Sampson et al. ${ }^{[137]}$. The efficacy of these immunotherapeutics has been limited, which is attributable to the overall poor immunogenicity of the CNS, heterogeneous expression of immune-targetable antigens, and tumor evolution over time ${ }^{[137,138]}$. Oncolytic viruses are also employed to selectively infect tumor cells - inducing virus-mediated cell death as well as promoting secondary immune response - and have shown promise ${ }^{[156]}$. For each of these strategies, clones with innate resistance or acquired resistance promote disease recurrence and progression ${ }^{[42]}$.

\section{FUTURE DIRECTIONS}

Overall, future treatments will need to address intratumoral heterogeneity, treatment escape mechanisms, and microenvironmental influences to circumnavigate disease progression and allow for the creation of standardized protocols for the treatment of recurrent and progressive GBM. Understanding the myriad reasons that lead to treatment failure in GBM, including the inability to obtain a complete resection, challenges of drug delivery and crossing the $\mathrm{BBB}$, limitations in clinical trial design and execution, intertumoral and intratumoral heterogeneity, reacquisition of stemness in GSCs, dynamics of the tumor microenvironment, metabolic adaptations, and the role of EVs in therapy resistance, are critical in developing targeted therapies. Lack of proper in vivo models, limited knowledge on drivers of progressive disease, resistance mechanisms, and treatment-induced molecular and genetic diversity have hindered the growth at the GBM therapeutic front. Liquid biopsy-based strategies could further help us understand tumor heterogeneity and the evolution of the genomic architecture of the tumor. This knowledge is essential for integrating precision diagnostics into personalized therapeutics. As such, multimodal treatment can be dynamic and tailored to the evolutionary landscape of the tumor, thus minimizing the development of resistance and ensuring a durable treatment response.

\section{CONCLUSION}

Personalized therapeutic strategies complementing the evolving molecular landscape of the tumor are essential to overcome recurrence and resistance. Multidimensional approaches such as combinations of chemotherapy, radiation, and immunotherapy are critical to curtailing the cycle of therapy and resistance. Understanding the dynamic role of EVs in enhancing resistance to therapy can provide novel therapeutic targets. Targeting EV-mediated mechanisms of resistance might supplement the existing therapeutic modalities. Additionally, liquid biopsy-based monitoring can supplement therapeutic efforts by providing real-time insights into the emerging intratumoral heterogeneity of the tumor, over time and therapy. 


\section{DECLARATIONS}

\section{Authors' contributions}

Made substantial contributions to conception and design of the study and performed data analysis and interpretation; conceptualization, writing, reviewing and editing: Yekula A, Taylor A, Beecroft A, Kang KM, Small JL, Muralidharan K, Rosh Z, Carter BS, Balaj L

\section{Availability of data and materials}

Not applicable.

\section{Financial support and sponsorship}

This work was supported by grants (U01 CA230697) to Carter BS, Balaj L; (P01 CA069246) to Carter BS; (R01CA239078, R01 CA237500) to Carter BS, Balaj L.

\section{Conflicts of interest}

All authors declared that there are no conflicts of interest.

\section{Ethical approval and consent to participate}

Not applicable.

\section{Consent for publication}

Not applicable.

\section{Copyright}

(C) The Author(s) 2021.

\section{REFERENCES}

1. Wen PY, Weller M, Lee EQ, et al. Glioblastoma in adults: a society for neuro-oncology (SNO) and European society of neuro-oncology (EANO) consensus review on current management and future directions. Neuro Oncol 2020; doi: 10.1093/neuonc/noaa106.

2. Stupp R, Mason WP, van den Bent MJ. Radiotherapy plus concomitant and adjuvant temozolomide for glioblastoma. Oncol Times 2005;27:15-6.

3. Cohen MH. Food and drug administration drug approval summary: temozolomide plus radiation therapy for the treatment of newly diagnosed glioblastoma multiforme. Clin Cancer Res 2005;11:6767-71.

4. Noch EK, Ramakrishna R, Magge R. Challenges in the treatment of glioblastoma: multisystem mechanisms of therapeutic resistance. World Neurosurg 2018;116:505-17.

5. Ralte AM, Sharma MC, Karak AK, Mehta VS, Sarkar C. Clinicopathological features, MIB-1 labeling index and apoptotic index in recurrent astrocytic tumors. Pathol Oncol Res 2001;7:267-78.

6. Sarkar C, Ralte AM, Sharma MC, Mehta VS. Recurrent astrocytic tumours--a study of p53 immunoreactivity and malignant progression. Br J Neurosurg 2002;16:335-42.

7. Mallick S, Benson R, Hakim A, Rath GK. Management of glioblastoma after recurrence: a changing paradigm. J Egypt Natl Canc Inst 2016;28:199-210.

8. Gruber ML, Buster WP. Temozolomide in combination with Irinotecan for treatment of recurrent malignant glioma. Am J Clin Oncol 2004;27:33-8.

9. Ricklefs FL, Alayo Q, Krenzlin H, et al. Immune evasion mediated by PD-L1 on glioblastoma-derived extracellular vesicles. Sci $A d v$ 2018;4:eaar2766.

10. Szatanek R, Baj-Krzyworzeka M, Zimoch J, Lekka M, Siedlar M, Baran J. The methods of choice for extracellular vesicles (EVs) characterization. Int J Mol Sci 2017; 18:1153.

11. Kosgodage US, Uysal-Onganer P, MacLatchy A, et al. Peptidylarginine deiminases post-translationally deiminate prohibitin and modulate extracellular vesicle release and microRNAs in glioblastoma multiforme. Int J Mol Sci 2018;20.

12. Yekula A, Yekula A, Muralidharan K, Kang K, Carter BS, Balaj L. Extracellular vesicles in glioblastoma tumor microenvironment. Front Immunol 2019;10:3137.

13. Skog J, Würdinger T, van Rijn S, et al. Glioblastoma microvesicles transport RNA and proteins that promote tumour growth and provide diagnostic biomarkers. Nat Cell Biol 2008;10:1470-6.

14. Shankar GM, Balaj L, Stott SL, Nahed B, Carter BS. Liquid biopsy for brain tumors. Exp Rev Mol Diagn 2017;17:943-7.

15. Bark JM, Kulasinghe A, Chua B, Day BW, Punyadeera C. Circulating biomarkers in patients with glioblastoma. Br J Cancer 
2020;122:295-305.

16. Verhaak RGW, Hoadley KA, Purdom E, et al. Integrated genomic analysis identifies clinically relevant subtypes of glioblastoma characterized by abnormalities in PDGFRA, IDH1, EGFR, and NF1. Cancer Cell 2010;17:98-110.

17. Louis DN, Perry A, Reifenberger G, et al. The 2016 world health organization classification of tumors of the central nervous system: a summary. Acta Neuropathol 2016;131:803-20.

18. Wang $\mathrm{Q}, \mathrm{Hu} \mathrm{B}, \mathrm{Hu} \mathrm{X}$, et al. Tumor evolution of glioma-intrinsic gene expression subtypes associates with immunological changes in the microenvironment. Cancer Cell 2017;32:42-56.e6.

19. Young RM, Jamshidi A, Davis G, Sherman JH. Current trends in the surgical management and treatment of adult glioblastoma. Ann Transl Med 2015;3:121.

20. Neftel C, Laffy J, Filbin MG, et al. An integrative model of cellular states, plasticity, and genetics for glioblastoma. Cell 2019;178:83549.e21.

21. Kim H, Zheng S, Amini SS, et al. Whole-genome and multisector exome sequencing of primary and post-treatment glioblastoma reveals patterns of tumor evolution. Genome Res 2015;25:316-27.

22. Johnson BE, Mazor T, Hong C, et al. Mutational analysis reveals the origin and therapy-driven evolution of recurrent glioma. Science 2014;343:189-93.

23. Wang J, Cazzato E, Ladewig E, et al. Clonal evolution of glioblastoma under therapy. Nat Genet 2016;48:768-76.

24. Mazor T, Pankov A, Johnson BE, et al. DNA methylation and somatic mutations converge on the cell cycle and define similar evolutionary histories in brain tumors. Cancer Cell 2015;28:307-17.

25. Nowell PC. The clonal evolution of tumor cell populations. Science 1976;194:23-8.

26. Reinartz R, Wang S, Kebir S, et al. Functional subclone profiling for prediction of treatment-induced intratumor population shifts and discovery of rational drug combinations in human glioblastoma. Clin Cancer Res 2017;23:562-74.

27. Favero F, McGranahan N, Salm M, et al. Glioblastoma adaptation traced through decline of an IDH1 clonal driver and macro-evolution of a double-minute chromosome. Ann Oncol 2015;26:880-7.

28. Klughammer J, Kiesel B, Roetzer T, et al. The DNA methylation landscape of glioblastoma disease progression shows extensive heterogeneity in time and space. Nat Med 2018;24:1611-24.

29. Scorsetti M, Navarria P, Pessina F, et al. Multimodality therapy approaches, local and systemic treatment, compared with chemotherapy alone in recurrent glioblastoma. BMC Cancer 2015;15:486.

30. Singh SK, Hawkins C, Clarke ID, et al. Identification of human brain tumour initiating cells. Nature 2004;432:396-401.

31. Huang Z, Cheng L, Guryanova OA, Wu Q, Bao S. Cancer stem cells in glioblastoma - molecular signaling and therapeutic targeting. Protein Cell 2010;1:638-55.

32. Toda M. Glioma stem cells and immunotherapy for the treatment of malignant gliomas. ISRN Oncology 2013;2013:1-13.

33. Calabrese C, Poppleton H, Kocak M, et al. A perivascular niche for brain tumor stem cells. Cancer Cell 2007;11:69-82.

34. Meyer M, Reimand J, Lan X, et al. Single cell-derived clonal analysis of human glioblastoma links functional and genomic heterogeneity. Proc Natl Acad Sci U S A 2015;112:851-6.

35. Qazi MA, Vora P, Venugopal C, et al. A novel stem cell culture model of recurrent glioblastoma. J Neurooncol 2016;126:57-67.

36. Huang Q, Zhang QB, Dong J, et al. Glioma stem cells are more aggressive in recurrent tumors with malignant progression than in the primary tumor, and both can be maintained long-term in vitro. BMC Cancer 2008;8:304.

37. Jackson M, Hassiotou F, Nowak A. Glioblastoma stem-like cells: at the root of tumor recurrence and a therapeutic target. Carcinogenesis 2015;36:177-85.

38. Chen J, Li Y, Yu TS, et al. A restricted cell population propagates glioblastoma growth after chemotherapy. Nature 2012;488:522-6.

39. Liu G, Yuan X, Zeng Z, et al. Analysis of gene expression and chemoresistance of CD133+ cancer stem cells in glioblastoma. Mol Cancer 2006;5:67.

40. Bao $\mathrm{S}, \mathrm{Wu} \mathrm{Q}, \mathrm{McLendon} \mathrm{RE}$, et al. Glioma stem cells promote radioresistance by preferential activation of the DNA damage response. Nature 2006;444:756-60.

41. Osuka S, Sampetrean O, Shimizu T, et al. IGF1 receptor signaling regulates adaptive radioprotection in glioma stem cells. Stem Cells 2013;31:627-40.

42. Osuka S, Van Meir EG. Overcoming therapeutic resistance in glioblastoma: the way forward. J Clin Invest 2017;127:415-26.

43. Andersson ER, Lendahl U. Therapeutic modulation of Notch signalling - are we there yet? Nat Rev Drug Discov 2014;13:357-78.

44. Pandit R, Chen L, Götz J. The blood-brain barrier: physiology and strategies for drug delivery. Adv Drug Deliv Rev 2019; doi: 10.1016/ j.addr.2019.11.009.

45. Arvanitis CD, Ferraro GB, Jain RK. The blood-brain barrier and blood-tumour barrier in brain tumours and metastases. Nat Rev Cancer 2020;20:26-41.

46. Salaroglio IC, Abate C, Rolando B, et al. Validation of thiosemicarbazone compounds as P-glycoprotein inhibitors in human primary brain-blood barrier and glioblastoma stem cells. Mol Pharm 2019;16:3361-73.

47. Haar CP, Hebbar P, Wallace GC 4th, et al. Drug resistance in glioblastoma: a mini review. Neurochem Res 2012;37:1192-200.

48. Agarwal S, Hartz AMS, Elmquist WF, Bauer B. Breast cancer resistance protein and P-glycoprotein in brain cancer: two gatekeepers team up. Curr Pharm Des 2011;17:2793-802.

49. Agarwal S, Mittapalli RK, Zellmer DM, et al. Active efflux of Dasatinib from the brain limits efficacy against murine glioblastoma: broad implications for the clinical use of molecularly targeted agents. Mol Cancer Ther 2012;11:2183-92.

50. Hosokawa Y, Takahashi H, Inoue A, et al. Oct-3/4 modulates the drug-resistant phenotype of glioblastoma cells through expression of 
ATP binding cassette transporter G2. Biochim Biophys Acta 2015;1850:1197-205.

51. Becker CM, Oberoi RK, McFarren SJ, et al. Decreased affinity for efflux transporters increases brain penetrance and molecular targeting of a PI3K/mTOR inhibitor in a mouse model of glioblastoma. Neuro Oncol 2015;17:1210-9.

52. de Gooijer MC, de Vries NA, Buckle T, et al. Improved brain penetration and antitumor efficacy of temozolomide by inhibition of ABCB1 and ABCG2. Neoplasia 2018;20:710-20.

53. Durmus S, Sparidans RW, Wagenaar E, Beijnen JH, Schinkel AH. Oral availability and brain penetration of the B-RAFV600E inhibitor vemurafenib can be enhanced by the P-GLYCOprotein (ABCB1) and breast cancer resistance protein (ABCG2) inhibitor elacridar. Mol Pharm 2012;9:3236-45.

54. Mason WP. Blood-brain barrier-associated efflux transporters: a significant but underappreciated obstacle to drug development in glioblastoma. Neuro Oncol 2015;17:1181-2.

55. Broekman ML, Maas SLN, Abels ER, Mempel TR, Krichevsky AM, Breakefield XO. Multidimensional communication in the microenvirons of glioblastoma. Nat Rev Neurol 2018;14:482-95.

56. McGranahan T, Therkelsen KE, Ahmad S, Nagpal S. Current state of immunotherapy for treatment of glioblastoma. Curr Treat Options Oncol 2019;20:24.

57. Wolf A, Agnihotri S, Guha A. Targeting metabolic remodeling in glioblastoma multiforme. Oncotarget 2010;1:552-62.

58. Zhou W, Wahl DR. Metabolic abnormalities in glioblastoma and metabolic strategies to overcome treatment resistance. Cancers 2019;11:1231.

59. Masui K, Tanaka K, Ikegami S, et al. Glucose-dependent acetylation of Rictor promotes targeted cancer therapy resistance. Proc Natl Acad Sci U S A 2015;112:9406-11.

60. Schito L, Semenza GL. Hypoxia-inducible factors: master regulators of cancer progression. Trends Cancer Res 2016;2:758-70.

61. Anderson P, Kedersha N, Ivanov P. Stress granules, P-bodies and cancer. Biochim Biophys Acta 2015;1849:861-70.

62. Lebelo MT, Joubert AM, Visagie MH. Warburg effect and its role in tumourigenesis. Arch Pharm Res 2019;42:833-47.

63. Comerford KM, Wallace TJ, Karhausen J, Louis NA, Montalto MC, Colgan SP. Hypoxia-inducible factor-1-dependent regulation of the multidrug resistance (MDR1) gene. Cancer Res 2002;62:3387-94.

64. Vaupel P, Kelleher DK, Höckel M. Oxygen status of malignant tumors: pathogenesis of hypoxia and significance for tumor therapy. Semin Oncol 2001;28:29-35.

65. Spehalski EI, Lee JA, Peters C, Tofilon P, Camphausen K. The quiescent metabolic phenotype of glioma stem cells. J Proteomics Bioinform 2019;12:96-103.

66. Oliver L, Olivier C, Marhuenda FB, Campone M, Vallette FM. Hypoxia and the malignant glioma microenvironment: regulation and implications for therapy. Curr Mol Pharmacol 2009;2:263-84.

67. Epple LM, Dodd RD, Merz AL, et al. Induction of the unfolded protein response drives enhanced metabolism and chemoresistance in glioma cells. PLoS One 2013;8:e73267.

68. Ciccia A, Elledge SJ. The DNA damage response: making it safe to play with knives. Molecular Cell 2010;40:179-204.

69. Caracciolo D, Montesano M, Tagliaferri P, Tassone P. Alternative non-homologous end joining repair: a master regulator of genomic instability in cancer. Precis Cancer Med 2019;2:8.

70. Fu D, Calvo JA, Samson LD. Balancing repair and tolerance of DNA damage caused by alkylating agents. Nat Rev Cancer 2012;12:104-20.

71. Hegi ME, Diserens AC, Gorlia T, et al. MGMTGene silencing and benefit from temozolomide in glioblastoma. New Engl J Med 2005;352:997-1003.

72. Hunter C, Smith R, Cahill DP, et al. A hypermutation phenotype and somatic MSH6 mutations in recurrent human malignant gliomas after alkylator chemotherapy. Cancer Res 2006;66:3987-91.

73. Erasimus H, Gobin M, Niclou S, Van Dyck E. DNA repair mechanisms and their clinical impact in glioblastoma. Mutat Res Rev Mut Res 2016;769:19-35.

74. Lawrence JE, Bammert CE, Belton RJ, Rovin RA, Winn RJ. Targeting DNA repair mechanisms to treat glioblastoma. Adv DNA Rep 2015; doi: 10.5772/59632

75. Osswald M, Jung E, Sahm F, et al. Brain tumour cells interconnect to a functional and resistant network. Nat 2015;528:93-8.

76. Namee NM, O’Driscoll L. Extracellular vesicles and anti-cancer drug resistance. Biochim Biophys Acta Rev Cancer 2018;1870:123-36.

77. Ning K, Wang T, Sun X, et al. UCH-L1-containing exosomes mediate chemotherapeutic resistance transfer in breast cancer. J Surg Oncol 2017;115:932-40.

78. Bebawy M, Combes V, Lee E, et al. Membrane microparticles mediate transfer of P-glycoprotein to drug sensitive cancer cells. Leukemia 2009;23:1643-9.

79. Aung T, Chapuy B, Vogel D, et al. Exosomal evasion of humoral immunotherapy in aggressive B-cell lymphoma modulated by ATPbinding cassette transporter A3. Proc Natl Acad Sci U S A 2011;108:15336-41.

80. Ma X, Cai Y, He D, et al. Transient receptor potential channel TRPC5 is essential for P-glycoprotein induction in drug-resistant cancer cells. Proc Natl Acad Sci U S A 2012;109:16282-7.

81. Yang SJ, Wang DD, Li J, et al. Predictive role of GSTP1-containing exosomes in chemotherapy-resistant breast cancer. Gene 2017;623:5-14.

82. Dong H, Wang W, Chen R, et al. Exosome-mediated transfer of lncRNA SNHG14 promotes trastuzumab chemoresistance in breast cancer. Int J Oncol 2018; doi: 10.3892/ijo.2018.4467.

83. Jing C, Cao H, Qin X, et al. Exosome-mediated gefitinib resistance in lung cancer HCC827 cells via delivery of miR-21. Oncol Lett 
2018;15:9811-7.

84. Fu X, Liu M, Qu S, et al. Exosomal microRNA-32-5p induces multidrug resistance in hepatocellular carcinoma via the PI3K/Akt pathway. J Exp Clin Cancer Res 2018;37.

85. Liu T, Chen G, Sun D, et al. Exosomes containing miR-21 transfer the characteristic of cisplatin resistance by targeting PTEN and PDCD4 in oral squamous cell carcinoma. Acta Biochim Biophys Sin 2017;49:808-16.

86. Zhang S, Zhang Y, Qu J, et al. Exosomes promote cetuximab resistance via the PTEN/Akt pathway in colon cancer cells. Braz J Med Biol Res 2017;51:e6472.

87. Vella LJ, Behren A, Coleman B, Greening DW, Hill AF, Cebon J. Intercellular resistance to BRAF inhibition can be mediated by extracellular vesicle-associated PDGFR $\beta$. Neoplasia 2017;19:932-40.

88. Fornari F, Pollutri D, Patrizi C, et al. In hepatocellular carcinoma miR-221 modulates Sorafenib Resistance through Inhibition of caspase3-mediated apoptosis. Clin Cancer Res 2017;23:3953-65.

89. Xu CG, Yang MF, Ren YQ, Wu CH, Wang LQ. Exosomes mediated transfer of lncRNA UCA1 results in increased tamoxifen resistance in breast cancer cells. Eur Rev Med Pharmacol Sci 2016;20:4362-8.

90. McCubrey JA, Abrams SL, Fitzgerald TL, et al. Roles of signaling pathways in drug resistance, cancer initiating cells and cancer progression and metastasis. Adv Biol Regul 2015;57:75-101.

91. Zheng P, Luo Q, Wang W, et al. Tumor-associated macrophages-derived exosomes promote the migration of gastric cancer cells by transfer of functional Apolipoprotein E. Cell Death Dis 2018;9:434.

92. Qin X, Yu S, Zhou L, et al. Cisplatin-resistant lung cancer cell-derived exosomes increase cisplatin resistance of recipient cells in exosomal miR-100-5p-dependent manner. Int J Nanomedicine 2017;12:3721-33.

93. Hu YB, Yan C, Mu L, et al. Exosomal Wnt-induced dedifferentiation of colorectal cancer cells contributes to chemotherapy resistance. Oncogene 2019;38:1951-65.

94. Ozawa PMM, Alkhilaiwi F, Cavalli IJ, Malheiros D, de Souza Fonseca Ribeiro EM, Cavalli LR. Extracellular vesicles from triplenegative breast cancer cells promote proliferation and drug resistance in non-tumorigenic breast cells. Breast Cancer Res Treat 2018;172:713-23.

95. Fatima F, Nawaz M. Stem cell-derived exosomes: roles in stromal remodeling, tumor progression, and cancer immunotherapy. Chin $J$ Cancer 2015;34:541-53.

96. Lobb RJ, van Amerongen R, Wiegmans A, Ham S, Larsen JE, Möller A. Exosomes derived from mesenchymal non-small cell lung cancer cells promote chemoresistance. Int J Cancer 2017;141:614-20.

97. Crow J, Atay S, Banskota S, Artale B, Schmitt S, Godwin AK. Exosomes as mediators of platinum resistance in ovarian cancer. Oncotarget 2017;8:11917-36.

98. Santos JC, Lima N da S, Sarian LO, Matheu A, Ribeiro ML, Derchain SFM. Exosome-mediated breast cancer chemoresistance via miR155 transfer. Sci Rep 2018;8:829.

99. Wang M, Qiu R, Yu S, et al. Paclitaxel resistant gastric cancer MGC 803 cells promote epithelial to mesenchymal transition and chemoresistance in paclitaxel sensitive cells via exosomal delivery of miR 155 5p. Int J Oncol 2018; doi: 10.3892/ijo.2018.4601.

100. Garnier D, Meehan B, Kislinger T, et al. Divergent evolution of temozolomide resistance in glioblastoma stem cells is reflected in extracellular vesicles and coupled with radiosensitization. Neuro Oncol 2018;20:236-48.

101. Baulch JE, Geidzinski E, Tran KK, Yu L, Zhou Y-H, Limoli CL. Irradiation of primary human gliomas triggers dynamic and aggressive survival responses involving microvesicle signaling. Environ Mol Mutagen 2016;57:405-15.

102. Zhang Z, Xu J, Chen Z, et al. Transfer of microRNA via macrophage-derived extracellular vesicles promotes proneural-to-mesenchymal transition in glioma stem cells. Cancer Immunol Res 2020; doi: 10.1158/2326-6066.CIR-19-0759.

103. Yue X, Lan F, Xia T. Hypoxic glioma cell-secreted exosomal miR-301a activates Wnt/ $\beta$-catenin signaling and promotes radiation resistance by targeting TCEAL7. Mol Ther 2019;27:1939-49.

104. Zeng AL, Yan W, Liu YW, et al. Tumour exosomes from cells harbouring PTPRZ1-MET fusion contribute to a malignant phenotype and temozolomide chemoresistance in glioblastoma. Oncogene 2017;36:5369-81.

105. Pavlyukov MS, Yu H, Bastola S, et al. Apoptotic cell-derived extracellular vesicles promote malignancy of glioblastoma via intercellular transfer of splicing factors. Cancer Cell 2018;34:119-35.e10.

106. Ramakrishnan V, Xu B, Akers J, et al. Radiation-induced extracellular vesicle (EV) release of miR-603 promotes IGF1-mediated stem cell state in glioblastomas. E Bio Med 2020;55:102736.

107. Dai X, Liao K, Zhuang Z, et al. AHIF promotes glioblastoma progression and radioresistance via exosomes. Int J Oncol 2019;54:261-70.

108. André-Grégoire G, Bidère N, Gavard J. Temozolomide affects extracellular vesicles released by glioblastoma cells. Biochimie 2018;155:11-5.

109. Yu T, Wang X, Zhi T, et al. Delivery of MGMT mRNA to glioma cells by reactive astrocyte-derived exosomes confers a temozolomide resistance phenotype. Cancer Lett 2018;433:210-20.

110. Shao H, Chung J, Lee K, et al. Chip-based analysis of exosomal mRNA mediating drug resistance in glioblastoma. Nat Commun 2015;6:6999.

111. Zhang Z, Yin J, Lu C, Wei Y, Zeng A, You Y. Exosomal transfer of long non-coding RNA SBF2-AS1 enhances chemoresistance to temozolomide in glioblastoma. J Exp Clin Cancer Res 2019;38:166.

112. Yin J, Zeng A, Zhang Z, Shi Z, Yan W, You Y. Exosomal transfer of miR-1238 contributes to temozolomide-resistance in glioblastoma. $E$ Bio Med 2019;42:238-51.

113. Mrowczynski OD, Madhankumar AB, Sundstrom JM, et al. Exosomes impact survival to radiation exposure in cell line models of 
nervous system cancer. Oncotarget 2018;9:36083-101.

114. Munoz JL, Walker ND, Mareedu S, et al. Cycling quiescence in temozolomide resistant glioblastoma cells is partly explained by microRNA-93 and -193-mediated decrease of cyclin D. Front Pharmacol 2019;10:134.

115. Richards KE, Zeleniak AE, Fishel ML, Wu J, Littlepage LE, Hill R. Cancer-associated fibroblast exosomes regulate survival and proliferation of pancreatic cancer cells. Oncogene 2017;36:1770-8.

116. Suklabaidya S, Dash P, Senapati S. Pancreatic fibroblast exosomes regulate survival of cancer cells. Oncogene 2017;36:3648-9.

117. Hu Y, Yan C, Mu L, et al. Fibroblast-derived exosomes contribute to chemoresistance through priming cancer stem cells in colorectal cancer. PLoS One 2015;10:e0125625.

118. Yeung CLA, Au Yeung CL, Co NN, et al. Exosomal transfer of stroma-derived miR21 confers paclitaxel resistance in ovarian cancer cells through targeting APAF1. Nat Commun 2016;7.

119. Zheng P, Chen L, Yuan X, et al. Exosomal transfer of tumor-associated macrophage-derived miR-21 confers cisplatin resistance in gastric cancer cells. J Exp Clin Cancer Res 2017;36:53.

120. Challagundla KB, Wise PM, Neviani P, et al. Exosome-mediated transfer of microRNAs within the tumor microenvironment and neuroblastoma resistance to chemotherapy. J Nat Cancer Inst 2015;107:djv135.

121. Chuang HY, Su YK, Liu HW, et al. Preclinical evidence of STAT3 inhibitor pacritinib overcoming temozolomide resistance via downregulating miR-21-enriched exosomes from M2 glioblastoma-associated macrophages. J Clin Med 2019;8:959.

122. Safaei R. Abnormal lysosomal trafficking and enhanced exosomal export of cisplatin in drug-resistant human ovarian carcinoma cells. Mol Cancer Ther 2005;4:1595-604.

123. Muralidharan-Chari V, Kohan HG, Asimakopoulos AG, et al. Microvesicle removal of anticancer drugs contributes to drug resistance in human pancreatic cancer cells. Oncotarget 2016;7:50365-79.

124. Samuel P, Fabbri M, Carter DRF. Mechanisms of drug resistance in cancer: the role of extracellular vesicles. Proteomics $2017 ; 17$.

125. Ifergan I, Scheffer GL, Assaraf YG. Novel extracellular vesicles mediate an ABCG2-dependent anticancer drug sequestration and resistance. Cancer Res 2005;65:10952-8.

126. Ciravolo V, Huber V, Ghedini GC, et al. Potential role of HER2-overexpressing exosomes in countering trastuzumab-based therapy. $J$ Cell Physiol 2012;227:658-67.

127. Simon T, Pinioti S, Schellenberger P, et al. Shedding of bevacizumab in tumour cells-derived extracellular vesicles as a new therapeutic escape mechanism in glioblastoma. Mol Cancer 2018;17:132.

128. Panagopoulos K, Cross-Knorr S, Dillard C, et al. Reversal of chemosensitivity and induction of cell malignancy of a non-malignant prostate cancer cell line upon extracellular vesicle exposure. Mol Cancer 2013;12:118.

129. Yuwen DL, Sheng BB, Liu J, Wenyu W, Shu YQ. MiR-146a-5p level in serum exosomes predicts therapeutic effect of cisplatin in nonsmall cell lung cancer. Eur Rev Med Pharmacol Sci 2017;21:2650-8.

130. Lv J, Chen Y, Zhou G, et al. Liquid biopsy tracking during sequential chemo-radiotherapy identifies distinct prognostic phenotypes in nasopharyngeal carcinoma. Nat Commun 2019;10.

131. Russo A, De Miguel Perez D, Gunasekaran M, et al. Liquid biopsy tracking of lung tumor evolutions over time. Expert Rev Mol Diagn 2019;19:1099-108.

132. Miller AM, Shah RH, Pentsova EI, et al. Tracking tumour evolution in glioma through liquid biopsies of cerebrospinal fluid. Nature 2019;565:654-8

133. Martinez VG, O'Neill S, Salimu J, et al. Resistance to HER2-targeted anti-cancer drugs is associated with immune evasion in cancer cells and their derived extracellular vesicles. Oncoimmunology 2017;6:e1362530.

134. Wei F, Ma C, Zhou T, et al. Exosomes derived from gemcitabine-resistant cells transfer malignant phenotypic traits via delivery of miRNA-222-3p. Mol Cancer 2017;16:132.

135. Zaika AI, Slade N, Erster SH, et al. $\Delta \mathrm{Np} 73$, a dominant-negative inhibitor of wild-type p53 and TAp73, is up-regulated in human tumors. $J$ Exp Med 2002;196:765-80.

136. Hou LC, Veeravagu A, Hsu AR, Tse VCK. Recurrent glioblastoma multiforme: a review of natural history and management options. Neurosurg Focus 2006;20:E5.

137. Sampson JH, Gunn MD, Fecci PE, Ashley DM. Brain immunology and immunotherapy in brain tumours. Nat Rev Cancer 2020;20:12-25.

138. Qazi MA, Vora P, Venugopal C, et al. Intratumoral heterogeneity: pathways to treatment resistance and relapse in human glioblastoma. Ann Oncol 2017;28:1448-56.

139. Yong RL, Wu T, Mihatov N, et al. Residual tumor volume and patient survival following reoperation for recurrent glioblastoma. $J$ Neurosurg 2014;121:802-9.

140. Ringel F, Pape H, Sabel M, et al. Clinical benefit from resection of recurrent glioblastomas: results of a multicenter study including 503 patients with recurrent glioblastomas undergoing surgical resection. Neuro Oncol 2016;18:96-104.

141. Brem H, Piantadosi S, Burger PC, et al. Placebo-controlled trial of safety and efficacy of intraoperative controlled delivery by biodegradable polymers of chemotherapy for recurrent gliomas. The Polymer-brain Tumor Treatment Group. Lancet 1995;345:1008-12.

142. Robertson CA, Hawkins Evans D, Abrahamse H. Photodynamic therapy (PDT): a short review on cellular mechanisms and cancer research applications for PDT. J Photochem Photobiol B 2009;96:1-8.

143. Elsaie MLT. Photodynamic therapy: new research. Nova Science; 2013.

144. Smith CJ, Fairres MJ, Myers CS, et al. Long-term outcome data from 121 patients treated with Gamma Knife stereotactic radiosurgery as salvage therapy for focally recurrent high-grade gliomas. J Radiosurg SBRT 2019;6:199-207.

145. Shrieve DC, Alexander E 3rd, Wen PY, et al. Comparison of stereotactic radiosurgery and brachytherapy in the treatment of recurrent 
glioblastoma multiforme. Neurosurgery 1995;36:275-82; discussion 282-4.

146. Bernstein M, Laperriere N, Glen J, Leung P, Thomason C, Landon AE. Brachytherapy for recurrent malignant astrocytoma. Int J Radiat Oncol Biol Phys 1994;30:1213-7.

147. Fogh SE, Andrews DW, Glass J, et al. Hypofractionated stereotactic radiation therapy: an effective therapy for recurrent high-grade gliomas. J Clin Oncol 2010;28:3048-53.

148. Ventero MP, Fuentes-Baile M, Quereda C, et al. Correction: radiotherapy resistance acquisition in glioblastoma. Role of SOCS1 and SOCS3. PLoS One 2019;14:e215714.

149. Friedman HS, Prados MD, Wen PY, et al. Bevacizumab alone and in combination with irinotecan in recurrent glioblastoma. J Clin Oncol 2009;27:4733-40

150. Kreisl TN, Kim L, Moore K, et al. Phase II trial of single-agent bevacizumab followed by bevacizumab plus irinotecan at tumor progression in recurrent glioblastoma. J Clin Oncol 2009;27:740-5.

151. Raizer JJ, Grimm S, Chamberlain MC, et al. A phase 2 trial of single-agent bevacizumab given in an every-3-week schedule for patients with recurrent high-grade gliomas. Cancer 2010;116:5297-305.

152. Winkler F, Osswald M, Wick W. Anti-angiogenics: their role in the treatment of glioblastoma. Oncol Res Treat 2018;41:181-6.

153. Ellis HP, Greenslade M, Powell B, Spiteri I, Sottoriva A, Kurian KM. Current challenges in glioblastoma: intratumour heterogeneity, residual disease, and models to predict disease recurrence. Front Oncol 2015;5:251.

154. Scribner E, Saut O, Province P, Bag A, Colin T, Fathallah-Shaykh HM. Effects of anti-angiogenesis on glioblastoma growth and migration: model to clinical predictions. PLoS One 2014;9:e115018.

155. Weller M, Cloughesy T, Perry JR, Wick W. Standards of care for treatment of recurrent glioblastoma-are we there yet? Neuro Oncol 2013;15:4-27.

156. Wollmann G, Ozduman K, van den Pol AN. Oncolytic virus therapy for glioblastoma multiforme: concepts and candidates. Cancer $J$ 2012;18:69-81. 